\title{
The Relationship between Genotype of Serotonin-transporter-linked Polymorphic Region (5-HTTLPR) and Migraine combined with Depression
}

\author{
Li Han, Zhenghe Yu and Hongjing Mao \\ Department of Psychosomatic, Mental Health Center, Zhejiang University School of Medicine, China
}

\begin{abstract}
The aim of this study is to explore whether there is an association between the genotype of serotonin-transporter-linked polymorphic region (5-HTTLPR) and migraine combined with depression.

One hundred and sixteen patients with migraine and depressive disorder (Group A) and 116 patients with simple migraine (Group B) admitted in Mental Health Center, Zhejiang University School of Medicine, China, from January 2018 to April 2020 were included in the present study. Polymerase chain reaction (PCR) and restriction fragment length polymorphism techniques were used for detection of 5-HTTLPR genotype. The 5-HTTLPR genotype and allele frequency between the two groups were compared. The results showed that there was no significant difference in $5-H T T L P R$ genotype (L/L, L/S and S/S) frequency and allele ( $\mathrm{S}$ and $\mathrm{L}$ ) frequency between Group $\mathrm{A}$ and Group B ( $\mathrm{p}=0.794$ and 0.491 , respectively). In conclusion, 5 -HTTLPR genotype might not be related to the onset of migraine combined with depression.
\end{abstract}

Key Words: Migraine, Depressive disorder, Serotonin-transporter-linked polymorphic region (5-HTTLPR).

How to cite this article: Han L, Yu Z, Mao H. The Relationship between Genotype of Serotonin-transporter-linked Polymorphic Region (5-HTTLPR) and Migraine combined with Depression. J Coll Physicians Surg Pak 2021; 31(08):998-1000.

Migraine is a chronic disease of neurovascular dysfunction, featuring primary and paroxysmal headache accompanied by autonomic neuro-system dysfunction. Migraine can occur repeatedly; and the main clinical manifestation is unilateral head with moderate to severe pulsating pain, which can cause cognitive, sleeping, emotional and physical disorders. Migraine is closely related to depression, and depression can bring huge humanistic and economic burden to migraine patients. ${ }^{1}$

5-HTTLPR is composed of a long allele (L) and a short allele (S) polymorphic insertion/deletion. The transcription ability of theS allele is significantly weaker than that of the L allele, resulting in low expression of 5-HT. At present, there are inconsistent reports on the role of 5-HTTLPR in the pathogenesis of depression. Some studies believed that 5-HTTLPR genotype had no significant effect on depression symptoms. ${ }^{2}$ However, studies had found that $S / S$ genotype of 5 -HTTLPR was associated with a 6.2 -fold onset of depression. ${ }^{3}$ At present, the relationship between the genotype of 5-HTTLPR and migraine combined with depression is still unclear.

Correspondence to: Hongjing Mao, Department of Psychosomatic, Mental Health Center, Zhejiang University

School of Medicine, China

E-mail: ut3w21@163.com

Received: December 28, 2020; Revised: March 19, 2021;

Accepted: April 09, 2021

DOI: https://doi.org/10.29271/jcpsp.2021.08.998
The aim of this study is to explore whether there is an association between the genotype of 5-HTTLPR and migraine combined with depression.

After the approval of the Hospital Ethics Committee, 116 patients with migraine and depressive disorder (Group A) and 116 patients with simple migraine (Group B), admitted in Mental Health Center, Zhejiang University School of Medicine, China, from January 2018 to April 2020 were included as the research subject. The inclusion criteria for Group A and Group B were that the patient met the diagnostic criteria for chronic migraine in the 2013 International classification of headache diseases (ICHD-III); age $\geq 18$ years; no history of drug abuse or preventive medication; no history of anxiety and depression before the onset of chronic migraine; the patient was in migraine attack stage; the Chinese Han nationality. Group A also needed to meet the diagnostic criteria of depression with Hamilton depression rating scale (HAMD) score $\geq 8$ points.

The exclusion criteria for Group A and Group B were that concomitant with serious systemic diseases (cardiovascular disease, acute infectious disease, blood disease, endocrine disease, allergy or hemolysis); secondary headache: headache caused by ear, nose pharyngeal or laryngeal disease, or intracranial disease; concomitant with other psychiatric diseases or taking other antipsychotic drugs, antiepileptic drugs; normal nervous system physical examination results, normal imaging examination results; pregnant or breastfeeding women. 
On the second day of admission, $3 \mathrm{~mL}$ morning fasting peripheral venous blood was sampled from patients in Group A and Group B for detection of 5-HTTLPR genotypes. The 5HTTLPR genotypes were determined using polymerase chain reaction (PCR) primers: 5'-GGCGTTGCCGCTCTGAATGC-3' (forward) and 5'-GAGGGACTGAGCTGGACAACCAC-3' (reverse). $P C R$ reaction conditions were that $95^{\circ} \mathrm{C}$ pre-denaturation for 5 minutes, $94^{\circ} \mathrm{C}$ denaturation for 30 seconds, annealing at $62^{\circ} \mathrm{C}$ for 30 seconds, extension at $72^{\circ} \mathrm{C}$ for one minute, 30 cycles in total. Finally, it was extended at $72{ }^{\circ} \mathrm{C}$ for 10 minutes. The PCR products were electrophoresed in $3 \%$ agarose for 30 mins, and each band was inspected under ultraviolet light, using 100 bp DNA ladder marker as standard control to check the size of each fragment. ${ }^{4}$ The 375 bp fragment was $S$ type. The 419 bp fragment was L-shaped. The corresponding 3 genotypes were homozygous L/L (419 bp, 419 bp), heterozygous S/L (375 bp, $419 \mathrm{bp}), \mathrm{S} / \mathrm{S}$ (375 bp, $375 \mathrm{bp})$.

SPSS version 25 has been used in data analysis for this study. The normality of all measurement data was checked by Kolmogorov-Smirnov test. Quantitative data were given as mean \pm S.D in accordance with normal distribution. Qualitative data were given as frequency $(n)$ and percentage (\%) and compared by Chi-squared test. The $p<0.05$ was statistically significant. In Group A, there were 52 males $(44.83 \%)$ and 64 females $(55.17 \%)$; the average age was $38.58 \pm 7.67$ years; the average duration of migraine was $4.18 \pm 0.61$ years. In Group B, there were 54 males (46.55\%) and 62 females (53.45\%); the average age was $38.10 \pm 7.57$ years; the average duration of migraine was $4.06 \pm 0.59$ years.

The distribution of genotypes in Group A and Group B, both conformed to Hardy-Weinberg equilibrium law, Group $A X^{2}=$ $0.357, p=0.837$; Group $B X^{2}=0.330, p=0.848$, indicating that both groups were representative of the population.

There was no significant difference in 5-HTTLPR genotype (L/L, $\mathrm{L} / \mathrm{S}$ and $\mathrm{S} / \mathrm{S}$ ) frequency and allele ( $\mathrm{S}$ and $\mathrm{L}$ ) frequency between Group A and Group B ( $p=0.794$ and 0.491 , respectively), as shown in Tablel.

Table I: Comparison of 5-HTTLPR genotype and allele frequency between the twogroups.

\begin{tabular}{|l|l|l|l|l|}
\hline \multicolumn{2}{|l|}{ Parameter } & $\begin{array}{l}\text { Group A } \\
\text { (n=116) }\end{array}$ & $\begin{array}{l}\text { Group B } \\
\text { (n=116) }\end{array}$ & p-value \\
\hline \multirow{2}{*}{$\begin{array}{l}\text { Genotype } \\
\text { frequency }\end{array}$} & L/L & $14(12.07)$ & $16(13.79)$ & \\
\cline { 2 - 4 }$[\mathrm{L}(\%)]$ & $\mathrm{L} / \mathrm{S}$ & $46(39.66)$ & $49(42.24)$ & \multirow{2}{*}{0.794} \\
\cline { 2 - 4 } $\begin{array}{l}\text { Allele } \\
\text { frequency } \\
\text { [n(\%)] }\end{array}$ & $\mathrm{S}$ & $156(48.28)$ & $51(43.97)$ & \\
\cline { 2 - 4 } & $\mathrm{L}$ & $74(31.90)$ & $151(65.09)$ & \multirow{2}{*}{0.491} \\
\hline
\end{tabular}

The 5-HTTLPR polymorphism may play an important role in this migraine disease, involving polygenic etiology of migraine, combined with aura. ${ }^{5}$ Studies have shown that 5-HTTLPR genotype may be related to the occurrence of coronary heart disease, combined with depression. ${ }^{6}$ This study showed that 5 HTTLPR genotype might not be related to the onset of migraine, combined with depression.
It can be seen that there are inconsistencies in the results of studies on the relationship between 5-HTTLPR gene polymorphism and depression. The reason may be that depression is a complex disease, and its occurrence can be affected by multiple susceptible genes, psychological, microscopic and macro-environmental factors. Each pathogenic gene alone may play a trivial role. Ethnic differences lead to the difference of the size of haploid domains between races. 5-HTTLPR gene polymorphisms may have different subtypes that affect transcriptional activity in races, so that the results of studies are difficult to be verified in other races. In addition, the sample size of this study is small, the detected polymorphic sites are few, and the statistical power is low, so there may be false positive results. In future, the sample size can be expanded, all polymorphic sites on 5-HTTLPR that may be related to depression can be detected with brain function imaging and other methods, and attention shall be paid to the influence of confounding factorssuch as environment, demographic characteristics, measurement tools and research design on the results, so as to further verify the relationship between 5-HTTLPR genotype and migraine, combined with depression.

\section{ACKNOWLEDGEMENT:}

This research was supported by Zhejiang Public Welfare Project (LGF20H090004); Hangzhou Science and Technology Commission Project (20191203B119); Hangzhou Science and Technology Commission Project (20180533B81); Hangzhou health and Family Planning Commission Project (2014A46).

\section{ETHICALAPPROVAL:}

This study was conducted after obtaining approval from the Hospital Ethics Committee of Mental Health Center, Zhejiang University School of Medicine, China.

\section{PATIENTS' CONSENT:}

Informed consents were obtained from the patients prior to inclusion in the study.

\section{CONFLICT OF INTEREST:}

The authors declared no conflict of interest.

\section{AUTHORS CONTRIBUTION:}

LH: Data analysis and interpretation, drafting of paper. ZY: Acquisition, analysis, interpretation of data.

HM: Revision of study, final approval of study for submission for publication.

\section{REFERENCES}

1. Alwhaibi M, Alhawassi TM. Humanistic and economic burden of depression and anxiety among adults with migraine: A systematic review. Depress Anxiety 2020; 37(11):1146-59. doi.org/10.1002/da.23063.

2. Thystrup CK, Vangkilde S, Ozenne B, Stenbæk DS. Severity of self-reported depressive symptoms in a healthy sample is modulated by trait Harm Avoidance, not by 5 -HTTLPR polymorphism. Psychiatry Res 2020; 291:113029. http:// doi.org/10.1016/ j.psychres.2020.113029.

3. Pooyan F, Gheysarzdeh A, Sadeghifard N, Kikhavani SL. Alleles of 5HTTLPR polymorphism of SLC6A4 gene in 
healthy people and patients with severe depression in Ilam province, Iran. Meta Gene 2020; 25: 100716. doi.org/ 10.1016/j.mgene.2020.100716.

4. Qiao J, Qian L, Geng D, Zhu X, Zhao H. Effects of the polymorphsim 5-HTTLPR on the hypersensitive C-reactive protein in patients with depression. Chin J Behav Med Brain Sci 2018, 27(1):17-21.

5. Marziniak M, Mossner R, Schmitt A, Lesch KP, Sommer C. A functional serotonin transporter gene is associated with migraine with aura. Neurology 2005 $764: 157-9$. doi.org/10. 1212/01.WNL.0000148597.52312.9E

6. Warnke K, Brandt J, Jörgens S, Arolt V, Beer K, Domschke $\mathrm{K}$, et al. Association of $5-\mathrm{HTTLPR} / \mathrm{rs} 25531$ with depressive symptoms in patients with coronary heart disease: $A$ prospective study. J Affective Disorders 2020; 277:531-9. doi.org/10.1016/j.jad.2020.08.046. 